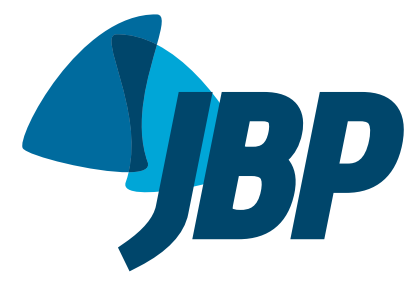

\title{
Lung transplantation: overall approach regarding its major aspects
}

\author{
Priscila Cilene León Bueno de Camargo', Ricardo Henrique de Oliveira Braga Teixeira', \\ Rafael Medeiros Carraro', Silvia Vidal Campos', José Eduardo Afonso Junior', \\ André Nathan Costa', Lucas Matos Fernandes'1, Luis Gustavo Abdalla', \\ Marcos Naoyuki Samano', Paulo Manuel Pêgo-Fernandes ${ }^{1,2}$
}

1. Grupo de Transplante Pulmonar Instituto do Coração, Hospital das Clínicas, Faculdade de Medicina, Universidade de São Paulo, São Paulo (SP) Brasil.

2. Departamento de Cirurgia Torácica, Faculdade de Medicina, Universidade de São Paulo, São Paulo (SP) Brasil.

Submitted: 30 April 2015

Accepted: 4 November 2015.

Study carried out by the Grupo de Transplante Pulmonar, Instituto do Coração, Hospital das Clínicas, Faculdade de Medicina, Universidade de São Paulo, São Paulo (SP) Brasil.

\begin{abstract}
Lung transplantation is a well-established treatment for patients with advanced lung disease. The evaluation of a candidate for transplantation is a complex task and involves a multidisciplinary team that follows the patient beyond the postoperative period. Currently, the mean time on the waiting list for lung transplantation in the state of São Paulo, Brazil, is approximately 18 months. For Brazil as a whole, data from the Brazilian Organ Transplant Association show that, in 2014, there were 67 lung transplants and 204 patients on the waiting list for lung transplantation. Lung transplantation is most often indicated in cases of COPD, cystic fibrosis, interstitial lung disease, non-cystic fibrosis bronchiectasis, and pulmonary hypertension. This comprehensive review aimed to address the major aspects of lung transplantation: indications, contraindications, evaluation of transplant candidates, evaluation of donor candidates, management of transplant recipients, and major complications. To that end, we based our research on the International Society for Heart and Lung Transplantation guidelines and on the protocols used by our Lung Transplant Group in the city of São Paulo, Brazil.
\end{abstract}

Keywords: Lung transplantation; Pulmonary disease, chronic obstructive; Cystic fibrosis; Respiratory tract infections; Pulmonary fibrosis; Hypertension, pulmonary.

\section{INTRODUCTION}

Lung transplantation is a treatment option for patients with advanced lung disease.

The evaluation of a lung transplant candidate is a task that involves a multidisciplinary team including pulmonologists, thoracic surgeons, infectious disease specialists, nurses, nutritionists, physiotherapists, psychologists, and social workers. When referring patients for an initial evaluation, pulmonologists should inform patients that lung transplantation is a treatment option that will be carefully analyzed by the lung transplant team, who will weigh the risks and benefits of the procedure.

In the state of São Paulo, Brazil, the mean time on the waiting list for lung transplantation is approximately 18 months. Although a donor lung is allocated to a given recipient primarily on the basis of $A B O$ blood compatibility, lung size compatibility is also important. Brazilian law does not provide prioritization criteria for patients on the waiting list for lung transplantation, the exception being acute graft failure within 30 days after the procedure.

Procedures include single lung transplantation (Figure 1) and bilateral lung transplantation, the latter being mandatory in cases of suppurative lung disease. Bilateral lung transplantation is the procedure of choice for patients with pulmonary hypertension because they are at an increased risk of primary graft dysfunction. Patients with severe right ventricular dysfunction, left ventricular dysfunction, or a combination of the two are candidates for heart-lung transplantation. However, procedures involving transplantation of a lung and another solid organ are not currently performed in Brazil.

Brain-dead donor organs are used in all of the aforementioned procedures. In exceptional cases (i.e., for pediatric recipients), living lobar lung transplantation can be performed, in which case there are two donors, each donating one lobe to the recipient.

For Brazil as a whole, data from the Brazilian Organ Transplant Association show that, in 2014, there were 67 lung transplants and 204 patients on the waiting list for lung transplantation. ${ }^{(1)}$

Lung transplantation is a well-established treatment that improves survival and quality of life in patients with advanced chronic lung disease. The present study is a comprehensive review of the major aspects of lung transplantation, including indications, contraindications, evaluation of transplant candidates, evaluation of donor candidates, management of transplant recipients, and major complications.

In 2014, the International Society for Heart and Lung Transplantation (ISHLT) met in order to update consensus guidelines on indications and contraindications for lung transplantation, as well as on candidate and donor selection. (2) The criteria described below are based on the 2014 ISHLT guidelines and on the protocols used by our Lung Transplant Group at the University of São Paulo School of Medicine Hospital das Clínicas Heart Institute, in the city of São Paulo, Brazil. 
Referring patients for lung transplant evaluation is an onerous task, and many patients are referred too late for evaluation.

Although several factors should be taken into consideration when evaluating patients for lung transplantation, patients in whom short- and long-term post-transplant survival rates are estimated to be higher than $80 \%$ constitute ideal candidates. The number of donor lungs available for transplantation is not enough to meet the demand, waiting list mortality being approximately $22 \%$. The entire lung transplantation process (from the initial evaluation to postoperative follow-up, from which recipients are never discharged) is complex and costly; therefore, candidates should be carefully evaluated so that financial resources are not unnecessarily spent on cases in which lung transplantation is likely to fail and donor lungs are allocated to patients whose chances of survival are known to be higher.

By 2014, our group had performed 232 lung transplants, most of which had been performed in patients with COPD, cystic fibrosis, interstitial lung disease, or non-cystic fibrosis bronchiectasis. Data from the ISHLT show that 1-, 3-, and 5-year survival rates after lung transplantation are $82.0 \%, 66.7 \%$, and $55.3 \%$, respectively. For procedures performed by our group, the aforementioned rates are $71.0 \%$, $59.8 \%$, and $55.2 \%$, respectively.

\section{CONTRAINDICATIONS}

The contraindications below are based on the ISHLT guidelines and on the protocols used by our Lung Transplant Group.

\section{Absolute contraindications}

- A recent history of cancer is an absolute contraindication to lung transplantation, a disease-free period of 2 years being required in all cases except those of nonmelanoma skin cancer (if properly treated). A disease-free period of 5 years is recommended for most other cancer patients, including those with hematological malignancies, sarcoma, melanoma, kidney cancer, bladder cancer, or breast cancer. Certain histological types of cancer confer a high risk of recurrence regardless of treatment duration and should therefore be evaluated on a case-by-case basis.

- Significant dysfunction of another major organ (such as the heart, the liver, the kidneys, and the brain) is an absolute contraindication to lung transplantation unless a double organ transplant can be performed. As previously mentioned, procedures involving transplantation of a lung and another solid organ are not currently performed in Brazil.

- Untreated or untreatable coronary artery disease with suspected or confirmed ischemic cardiac dysfunction is an absolute contraindication to lung transplantation.

- Acute medical instability, including, but not limited to, sepsis, acute myocardial infarction, and liver failure, is an absolute contraindication to lung transplantation.

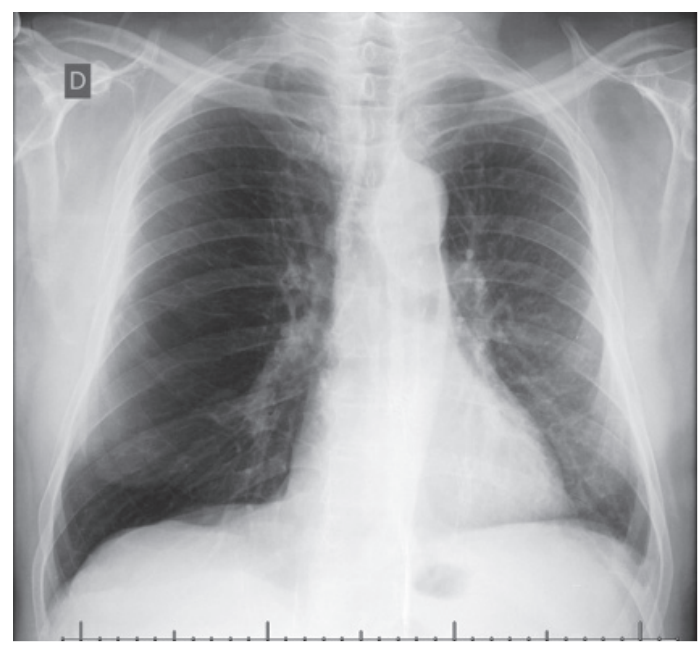

Figure 1. Chest X-ray of a left lung transplant recipient who underwent transplantation because of COPD.

- Untreatable bleeding diathesis is an absolute contraindication to lung transplantation.

- Poorly controlled chronic infection with highly virulent/resistant pathogens is an absolute contraindication to lung transplantation.

- Evidence of active infection with Mycobacterium tuberculosis is an absolute contraindication to lung transplantation.

- Chest wall or spinal column deformity that can lead to severe restrictive lung disease after transplantation is an absolute contraindication to lung transplantation.

- $\quad$ B BMI $\geq 35 \mathrm{~kg} / \mathrm{m}^{2}$ is an absolute contraindication to lung transplantation. At our facility, a BMI $\geq$ $30 \mathrm{~kg} / \mathrm{m}^{2}$ is an absolute contraindication to lung transplantation.

- Psychiatric or psychological conditions resulting in inability to cooperate with medical care or the health care team and in poor treatment adherence are absolute contraindications to lung transplantation.

- Inadequate social support is an absolute contraindication to lung transplantation.

- Poor functional status with low potential for rehabilitation is an absolute contraindication to lung transplantation. At our facility, lung transplant candidates undergo muscle strength assessment and the six-minute walk test, a six-minute walk distance (6MWD) of less than $200 \mathrm{~m}$ being a contraindication to the procedure. (3)

- Chemical abuse or dependency (including alcohol, tobacco, and illicit drug use/abuse) is an absolute contraindication to lung transplantation. At our facility, a minimum of six months of abstinence and complete cessation are required for patients undergoing lung transplantation.

\section{Relative contraindications}

- Being over 65 years of age and the presence of diminished psychological reserve, other relative contraindications, or a combination of the two are relative contraindications to lung transplantation, as is, in most cases, being over 75 years of age. At 
our facility, patients over 60 years of age are not placed on the waiting list for lung transplantation.

- $\quad A B M I \geq 30 \mathrm{~kg} / \mathrm{m}^{2}$ is a relative contraindication to lung transplantation. As previously mentioned, a BMI $\geq 30 \mathrm{~kg} / \mathrm{m}^{2}$ is an absolute contraindication to lung transplantation at our facility.

- Severe malnutrition is a relative contraindication to lung transplantation.

- Severe symptomatic osteoporosis is a relative contraindication to lung transplantation.

- Having undergone major thoracic surgery with pulmonary resection is a relative contraindication to lung transplantation.

- Being on mechanical ventilation, extracorporeal membrane oxygenation, or a combination of the two is a relative contraindication to lung transplantation. However, selected patients, without acute or chronic dysfunction of other organs, can undergo transplantation under these conditions.

- Colonization or infection with virulent pathogens or certain mycobacterial strains is a relative contraindication to lung transplantation.

- Active infection with $\mathrm{HBV}, \mathrm{HCV}$, or a combination of the two is a relative contraindication to lung transplantation. In some centers, patients infected with $\mathrm{HBV}, \mathrm{HCV}$, or both are considered for transplantation if there are no signs of cirrhosis or portal hypertension and if they are receiving appropriate treatment. At our facility, patients infected with HBV, HCV, or both are considered for transplantation if there are no signs of cirrhosis or portal hypertension and after they have received appropriate treatment and achieved an undetectable viral load.

- In some centers, patients with HIV infection are considered for transplantation if they have an undetectable viral load and adhere to treatment. At our facility, positive HIV serology is considered to be an absolute contraindication to lung transplantation.

- Infection with Burkholderia cenocepacia, B. gladioli, or Mycobacterium abscessus is considered to be a contraindication to lung transplantation in some centers. At our facility, postoperative complications in patients infected with any of the aforementioned bacteria have been only minor. Therefore, infection with $B$. cenocepacia, $B$. gladioli, or M. abscessus is not a contraindication to lung transplantation there.

- Atherosclerotic disease that is sufficiently advanced to pose a threat to lung transplant recipients is a relative contraindication to lung transplantation.

- Comorbidities such as systemic arterial hypertension, diabetes mellitus, gastroesophageal reflux disease, epilepsy, and peptic ulcer constitute a relative contraindication to lung transplantation and should be optimally treated prior to transplantation.

- Antiphospholipid syndrome is no contraindication to lung transplantation if it is not accompanied by heparin-induced thrombocytopenia.

- In patients with connective tissue disease, particularly in those with scleroderma, esophageal involvement is the major limitation to the success of lung transplantation because gastroesophageal reflux disease is a major risk factor for chronic allograft dysfunction.

\section{INDICATIONS}

In general, lung transplantation is indicated for patients who meet all of the following criteria:

- $\quad$ high (> 50\%) risk of 2-year mortality from lung disease if lung transplantation is not performed

- $\quad$ high (>90\%) probability of survival at 90 days after transplantation

- $\quad$ high (> 80\%) probability of survival at 5 years after transplantation, from a clinical standpoint, if the graft is in good condition

\section{Obstructive diseases}

Obstructive diseases include COPD and bronchiolitis obliterans.

Patients should be referred for evaluation in the following situations: progressive disease, despite optimal treatment; COPD that cannot be treated with lung volume reduction surgery or endoscopic lung volume reduction; a Body mass index, airflow Obstruction, Dyspnea, and Exercise capacity (BODE) index ${ }^{(4)}$ of 5-6; $\mathrm{PaCO}_{2}>50 \mathrm{mmHg}, \mathrm{PaO}_{2}<60 \mathrm{mmHg}$, or a combination of the two; and $\mathrm{FEV}_{1}<25 \%$ of predicted.

Patients should be placed on the waiting list for lung transplantation if they meet at least one of the following criteria: a BODE index $\geq 7 ; \mathrm{FEV}_{1}<15-20 \%$ of predicted; three or more severe exacerbations in the last year; one severe exacerbation with acute hypercapnic respiratory failure; and moderate to severe pulmonary hypertension.

\section{Suppurative diseases}

Suppurative diseases include cystic fibrosis, ciliary dyskinesia, and bronchiectasis.

Patients should be referred for evaluation in the following situations: $\mathrm{FEV}_{1}<30 \%$ of predicted, particularly in cases of rapid decline despite optimal treatment; 6MWD $<400 \mathrm{~m}$; development of pulmonary hypertension in the absence of hypoxemia secondary to exacerbation; and clinical worsening characterized by increased exacerbations accompanied by any of the following: acute respiratory failure requiring noninvasive ventilation; increased antimicrobial resistance and poor recovery from exacerbation; poor nutritional status despite supplementation; pneumothorax; or life-threatening hemoptysis that cannot be controlled with embolization.

Patients should be placed on the waiting list for lung transplantation if they meet at least one of the following criteria: chronic respiratory failure $\left(\mathrm{PaCO}_{2}>50 \mathrm{mmHg}\right.$, $\mathrm{PaO}_{2}<60 \mathrm{mmHg}$, or a combination of the two); need for noninvasive ventilation; pulmonary hypertension; frequent hospitalizations; rapid decline in lung function; and World Health Organization functional class IV.

In patients with bronchiectasis secondary to cystic fibrosis, the following should be taken into consideration: exocrine and endocrine pancreatic disease, 
which influences the choice of immunosuppressant (tacrolimus being more toxic to the pancreas than cyclosporine), and chronic sinus disease, given that colonization of the upper airways is a common cause of pulmonary infection after transplantation (sinusectomy is commonly indicated for lung transplant recipients).

\section{Interstitial diseases}

Interstitial diseases include fibrotic restrictive lung diseases, such as idiopathic pulmonary fibrosis, hypersensitivity pneumonitis, and nonspecific interstitial pneumonia.

Patients should be referred for evaluation in the following situations: histological or radiological evidence of usual interstitial pneumonitis or fibrotic nonspecific interstitial pneumonitis, independently of lung function; FVC $<80 \%$ of predicted or DLCO $<40 \%$ of predicted; dyspnea or functional limitation attributable to lung disease; need for supplemental oxygen, even if only during exercise; and, in cases of inflammatory interstitial lung disease (other than usual interstitial pneumonitis and fibrotic nonspecific interstitial pneumonitis), no symptom improvement, need for supplemental oxygen, functional improvement after appropriate treatment, or any combination of the three.

Patients should be placed on the waiting list for lung transplantation if they meet at least one of the following criteria: $\geq 10 \%$ decline in FVC at 6 months of follow-up; $\geq 15 \%$ decline in DLCO at 6 months of follow-up; desaturation $<88 \%$, 6MWD $<250 \mathrm{~m}$, or a $>50-\mathrm{m}$ decline in the 6MWD at 6 months of follow-up; pulmonary hypertension; or hospitalization because of functional deterioration, pneumothorax, or acute exacerbation.

\section{Vascular diseases \\ Vascular diseases include pulmonary arterial hypertension.}

Patients should be referred for evaluation in the following situations: New York Heart Association (NYHA) functional class III or IV despite optimal treatment; rapidly progressive disease; parenteral therapy use regardless of NYHA functional class (not available in Brazil); and confirmed or suspected pulmonary veno-occlusive disease or a diagnosis of pulmonary capillary hemangiomatosis.

Patients should be placed on the waiting list for lung transplantation if they meet at least one of the following criteria: NYHA functional class III or IV despite optimal treatment, including prostanoids (which are not yet widely available in Brazil); cardiac index $<2 \mathrm{~L} / \mathrm{min} /$ $\mathrm{m}^{2}$; mean right atrial pressure $>15 \mathrm{mmHg} ; 6 \mathrm{MWD}<$ $350 \mathrm{~m}$; or hemoptysis, pericardial effusion, or signs of right heart failure.

As previously mentioned, for lung or heart-lung transplantation referral, it is essential to determine whether the right ventricle is viable. This can be done by means of magnetic resonance imaging, myocardial scintigraphy, or both.

\section{Pediatric population}

Pediatric patients should be referred for evaluation in the same situations as should adult patients.
Certain particularities should be taken into account, primarily related to the size of the recipients, which can lead to a longer waiting time.

Treatment adherence is also important and tends to be lower in the pediatric population than in the adult population, poor treatment adherence increasing the risk of complications, including acute rejection and chronic allograft dysfunction. However, the success rates in the pediatric population are similar to those in the adult population. ${ }^{(5,6)}$

\section{Retransplantation}

The number of cases of lung transplant recipients who develop chronic graft failure and, consequently, chronic respiratory failure requiring retransplantation has increased worldwide.

\section{EVALUATION OF TRANSPLANT CANDIDATES}

Lung transplant candidates should be evaluated by a multidisciplinary team including pulmonologists, thoracic surgeons, infectious disease specialists, nurses, nutritionists, physiotherapists, psychologists, and social workers.

\section{Required tests}

- laboratory tests: complete blood count; coagulation profile; blood typing; urea; creatinine; sodium; potassium; ionized calcium; magnesium; total cholesterol, LDL cholesterol, and HDL cholesterol; triglycerides; total and direct bilirubin; total protein and albumin; lactate dehydrogenase; aspartate aminotransferase; alanine aminotransferase; alkaline phosphatase; gamma-glutamyltransferase; amylase; arterial blood gas analysis on room air; TSH and free thyroxine; serology for HIV, hepatitis A virus, HBV, $\mathrm{HCV}$, syphilis, Chagas disease, cytomegalovirus (CMV), toxoplasmosis, Epstein-Barr virus, and HSV; blood glucose; glycated hemoglobin; immune panel (after placement on the waiting list); urinalysis; creatinine clearance; and sputum examination (aerobic culture, AFB smear testing, AFB culture, screening for fungi, and fungal culture)

- full pulmonary function testing, including DLCO measurement

- imaging tests: posteroanterior and lateral chest X-rays; electrocardiography; HRCT; cranial CT; sinus CT (for patients with suppurative lung disease); quantitative lung perfusion radionuclide scanning; echocardiography; left heart catheterization (for patients over 40 years of age); right heart catheterization (for patients with echocardiographic signs of pulmonary hypertension); and bone densitometry

Patients with a history of gastroesophageal reflux disease or scleroderma should undergo $\mathrm{pH}$ monitoring and esophageal manometry.

Whenever necessary, patients should be evaluated by a gastroenterologist, a psychiatrist, a cardiologist, a nephrologist, a neurologist, a hematologist, a urologist, or a gynecologist. 
The decision to place a patient on the waiting list for lung transplantation is made by the multidisciplinary team on a case-by-case basis and is based on the results of the complete evaluation of the patient.

When a patient is placed on the waiting list for lung transplantation, an immune panel is requested in order to identify the presence of pre-transplant HLA class I and II antibodies. A > 10\% positive immune panel increases the risk of hyperacute rejection, and pre-transplant virtual crossmatch is therefore required. The immune panel should be repeated every 6 months. Blood transfusions can stimulate the formation of antiHLA antibodies. Therefore, if a patient on the waiting list for lung transplantation receives a blood product transfusion, the immune panel should be repeated.

\section{EVALUATION OF DONOR CANDIDATES}

Data from the Brazilian Organ Transplant Association show that, in 2014, Brazil had approximately 190.8 million inhabitants, with 14.2 actual donors per million population. Of those, only 0.4 per million population were actual lung donors.

Reasons for low organ donation rates include family refusal, cardiac arrest, and medical contraindications (poor donor quality).

The criteria for ideal and marginal donors are summarized below. Marginal donors meeting two or more of the criteria below should be excluded as candidates for organ donation.

Several studies have shown that the use of marginal donors does not affect outcome in the first year after lung transplantation. ${ }^{(7-9)}$

\section{Ideal donors}

- age $<55$ years

- smoking history < 20 pack-years

- no chest trauma

- mechanical ventilation $<48 \mathrm{~h}$

- no history of asthma

- no malignancies

- no bacteria in tracheal secretion or BAL fluid samples

- arterial blood gas analysis: $\mathrm{PaO}_{2}>300 \mathrm{mmHg}$ with a positive end-expiratory pressure of 5 $\mathrm{CmH}_{2} \mathrm{O}$ and an $\mathrm{FiO}_{2}$ of $100 \%$

- no chest X-ray findings of consolidation

- no bronchoscopic findings of airway secretions

Marginal donors (should be excluded as donor candidates if they meet two or more of the criteria below)

- $\quad$ age > 55 years

- $\quad$ smoking history $>20$ pack-years

- chest trauma

- mechanical ventilation $>48 \mathrm{~h}$

- personal history of asthma

- central nervous system tumor

- $\quad$ presence of bacteria in tracheal secretion or BAL fluid samples
- $\quad$ arterial blood gas analysis: $\mathrm{PaO}_{2}<300 \mathrm{mmHg}$ with a positive end-expiratory pressure of 5 $\mathrm{CmH}_{2} \mathrm{O}$ and an $\mathrm{FiO}_{2}$ of $100 \%$

- $\quad$ abnormal chest X-ray findings

- bronchoscopic findings of airway secretions

\section{MANAGEMENT OF TRANSPLANT RECIPIENTS}

Below, we describe how lung transplant recipients are managed by our Lung Transplant Group, our protocols being based on the ISHLT guidelines and on criteria established in the literature.

\section{Immunosuppression}

Immunosuppression is induced with a corticosteroid (methylprednisolone $500 \mathrm{mg}$ i.v.) and an anti-IL-2 receptor monoclonal antibody (basiliximab $20 \mathrm{mg}$ i.v.), both of which are administered at induction of anesthesia.

Immunosuppression is maintained with concomitant use of a calcineurin inhibitor (cyclosporine or tacrolimus), a cell proliferation inhibitor (azathioprine or mycophenolate), and a corticosteroid (prednisone). In some situations, mammalian target of rapamycin inhibitors (sirolimus or everolimus) can be used in association with the aforementioned treatment regimen or replace any of the aforementioned drugs, although not before postoperative month 3, because of the high risk of anastomotic dehiscence.

\section{Prophylaxis}

\section{Bacterial infections}

The antibiotics used in the intraoperative and immediate postoperative periods should be chosen on the basis of the underlying lung disease.

In smear-negative cases of nonsuppurative lung disease, the drug of choice is cefepime, which should be maintained until postoperative day 14 . This treatment regimen can be modified on the basis of the results of cultures of specimens from the donor and the recipient (including donor blood, donor BAL fluid, and bronchial secretions from the donor and the recipient in the intraoperative period) or on the clinical status of the recipient.

In patients with suppurative lung disease, the treatment regimen is based on previous culture and antibiogram results.

\section{Viral infections}

Prophylaxis for viral infections includes prophylaxis for HSV and CMV infection. In cases in which donors and recipients are seronegative for CMV, recipients should receive prophylaxis for HSV infection (with oral acyclovir for 3 months). In other cases, recipients should receive prophylaxis with intravenous ganciclovir for 3 months. Seronegative patients receiving organs from seropositive donors-a group of lung transplant recipients who are at an increased risk of viral 
reactivation-should receive prophylaxis with oral valganciclovir for 6 months.

\section{Fungal infections}

Prophylaxis for fungal infections includes prophylaxis for infection with Aspergillus spp., Candida spp., and Pneumocystis jirovecii. Prophylaxis for infection with Aspergillus spp. includes inhaled amphotericin (10 $\mathrm{mg}$ twice daily) and itraconazole (400 mg/day for 3 months). Prophylaxis for infection with Candida spp. includes nystatin oral suspension (for 3 months). Prophylaxis for infection with $P$. jirovecii includes trimethoprim-sulfamethoxazole (400/80 mg per day indefinitely).

\section{MAJOR COMPLICATIONS}

The major complications of lung transplantation are described below.

\section{Primary graft dysfunction}

Similar to ARDS, primary graft dysfunction is defined as a $\mathrm{PaO}_{2} / \mathrm{FiO}_{2}$ ratio of $<300$ and radiological infiltrates within the first $72 \mathrm{~h}$ after lung transplantation.

Major risk factors for primary graft dysfunction include the use of cardiopulmonary bypass during surgery, a previous diagnosis of idiopathic pulmonary fibrosis, a previous diagnosis of pulmonary arterial hypertension, significant donor smoking history, and a high BMI. ${ }^{(10,11)}$

The management of primary graft dysfunction is similar to that of ARDS, including lung-protective ventilation strategies and general clinical support.

\section{Acute rejection}

There are two types of acute rejection: acute cellular rejection, which is the more common of the two and is characterized by perivascular and interstitial mononuclear cell infiltrates; and acute humoral rejection, which is also referred to as acute antibody-mediated rejection. Humoral rejection is more closely related to hyperacute rejection, which occurs immediately after the surgical procedure; however, it can occur later, with de novo donor-specific antibody formation leading to endothelial cell damage and, consequently, pulmonary capillaritis. ${ }^{(12)}$

Active surveillance for acute cellular rejection is performed by means of transbronchial biopsy, independently of patient symptoms, during the first year after lung transplantation. In addition, in the presence of clinical worsening or functional loss, acute cellular rejection is a differential diagnosis for infections and other complications.

The treatment of acute cellular rejection varies according to the degree of impairment and includes immunosuppressive dose adjustment, corticosteroid pulse therapy and, in selected cases, antithymocyte globulin.

Humoral rejection is treated with plasmapheresis (in order to remove donor-specific antibodies) and polyclonal immunoglobulin.

\section{Chronic allograft dysfunction}

It is currently known that there are different phenotypes of chronic allograft dysfunction, the most common being bronchiolitis obliterans syndrome (Figure 2), which manifests as a progressive decline in $\mathrm{FEV}_{1}$ from baseline that cannot be attributed to acute rejection, infection, or bronchial stenosis. Major risk factors for chronic allograft dysfunction include previous viral infections, gastroesophageal reflux disease, and a history of acute rejection.

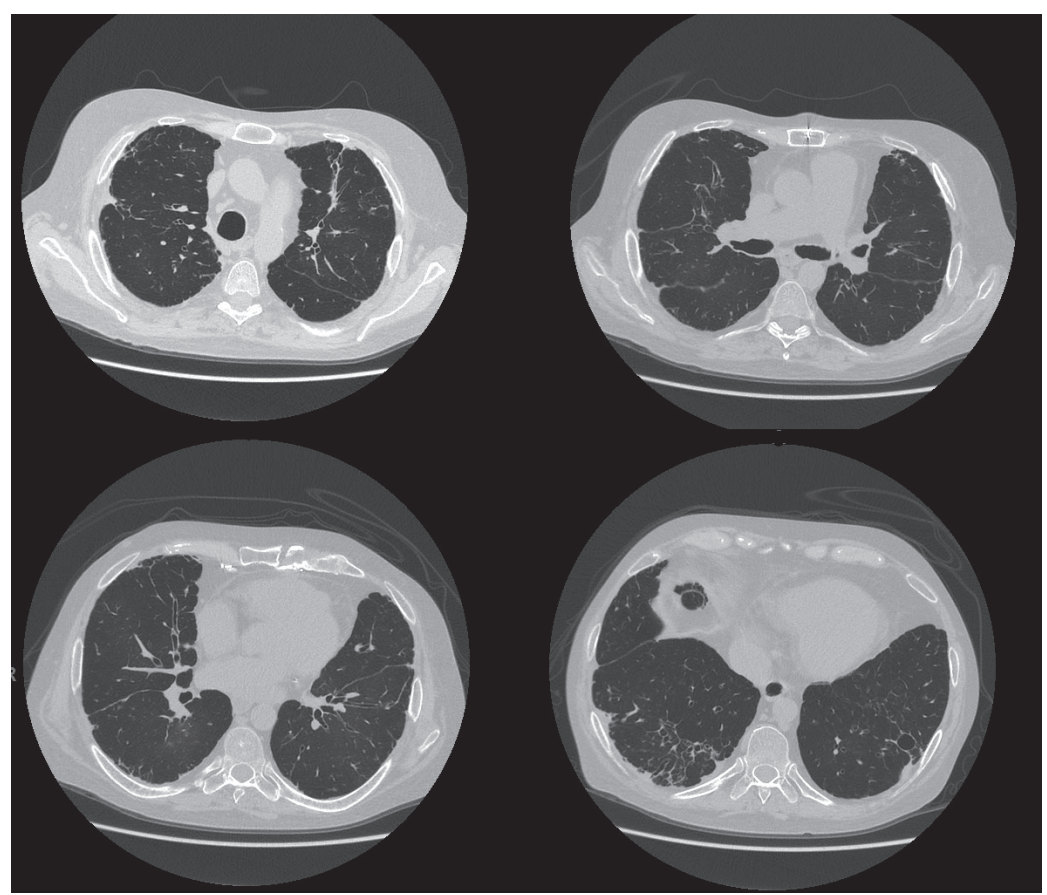

Figure 2. Chest CT scan of a patient with bronchiolitis obliterans syndrome. 
Other, less common, phenotypes have been described, including chronic allograft dysfunction characterized by restrictive ventilatory impairment and acute fibrinoid organizing pneumonia, the prognosis of which is worse than that of bronchiolitis obliterans syndrome. ${ }^{(13-15)}$

The management of chronic allograft dysfunction includes optimization of immunosuppression, removal of risk factors (such as gastroesophageal reflux disease), and, in cases that are more severe, retransplantation.

\section{Infections}

Lung transplant recipients are at risk of infection in the postoperative period. However, the prevalence of pathogens depends on the time elapsed since transplantation. (16) Until postoperative month 1, the most common infections are those related to the surgical procedure, the donor, or the recipient. From postoperative month 1 to postoperative month 6 , activation of latent infections (such as CMV infection and tuberculosis) is common. After postoperative month 6 , the prevalence of community-acquired infections (pneumonia and urinary tract infection) increases.

\section{Malignancies}

Patients receiving immunosuppressants are at an increased risk of malignancies. The most common types of malignancies are skin cancer and lymphoproliferative disorders. There is a relationship between the development of lymphoma and Epstein-Barr virus infection; recurrent CMV infection is a risk factor for the disease.

\section{Surgical complications}

Surgical complications occur in approximately $27 \%$ of cases and include dehiscence, necrosis, and bronchial anastomotic stenosis. Vascular complications such as venous stenosis are rare, occurring in $1-2 \%$ of cases.

Paralytic ileus is the most common abdominal complication, occurring in 30-50\% of patients. Gastroparesis, acute cholecystitis, and intestinal perforation can also occur, the mortality from intestinal perforation being high. ${ }^{(17-19)}$

\section{REFERENCES}

1. Associação Brasileira de Transplante de Órgãos [homepage on the Internet]. São Paulo: ABTO [cited 2015 Apr 30]. Registro Brasileiro de Transplantes 2014. Dimensionamento dos transplantes no Brasil e em cada estado (2007-2014). [Adobe Acrobat document, 98p.]. Available from: http://www.abto.org.br/abtov03/Upload/file/ RBT/2014/rbt2014-lib.pdf

2. Weill D, Benden C, Corris PA., Dark JH, Davis RD, Keshavjee S, et al. A consensus document for the selection of lung transplant candidates: 2014--an update from the Pulmonary Transplantation Council of the International Society for Heart and Lung Transplantation. J Heart Lung Transplant. 2015;34(1):1-15. http://dx.doi.org/10.1016/]. healun.2014.06.014

3. Martinu T, Babyak MA, O'Connell CF, Carney RM, Trulock EP, Davis $\mathrm{RD}$, et al. Baseline 6-min walk distance predicts survival in lung transplant candidates. Am J Transplant. 2008;8(7):1498-505. http:// dx.doi.org/10.1111/j.1600-6143.2008.02264.x

4. Casanova C, de Torres JP, Aguirre-Jaíme A, Pinto-Plata V, Marin JM Cordoba $\mathrm{E}$, et al. The progression of chronic obstructive pulmonary disease is heterogeneous: the experience of the BODE cohort. Am J Respir Crit Care Med. 2011;184(9):1015-21. http://dx.do org/10.1164/rccm.201105-08310C

5. Camargo PC, Pato EZ, Campos SV, Afonso JE Jr, Carraro RM, Costa $\mathrm{AN}$, et al. Pediatric lung transplantation: 10 years of experience. Clinics (Sao Paulo). 2014;69 Suppl 1:51-4. http://dx.doi.org/10.6061/ clinics/2014(Sup01)10

6. Mendeloff EN. The history of pediatric heart and lung transplantation. Pediatr Transplant. 2002;6(4):270-9. http://dx.doi.org/10.1034//.13993046.2002.00217.x

7. Pêgo-Fernandes $\mathrm{PM}$, Samano MN, Fiorelli Al, Fernandes LM, Camargo SM, Xavier AM, et al. Recommendations for the use of extended criteria donors in lung transplantation. Transplant Proc. 2011;43(1):216-9. http://dx.doi.org/10.1016/j. transproceed.2010.12.050

8. Botha P, Trivedi D, Weir CJ, Searl CP, Corris PA, Dark JH, et al. Extended donor criteria in lung transplantation: Impact on organ allocation. J Thorac Cardiovasc Surg. 2006;131(5):1154-60. http:// dx.doi.org/10.1016/j.jtcvs.2005.12.037

9. Bhorade SM, Vigneswaran W, McCabe MA, Garrity ER. Liberalization of donor criteria may expand the donor pool without adverse consequence in lung transplantation. J Heart Lung Transplant. 2000;19(12):1199204. http://dx.doi.org/10.1016/S1053-2498(00)00215-1
10. Samano MN, Fernandes LM, Baranauskas JC, Correia AT, Afonso $\mathrm{JE} \mathrm{Jr}$, Teixeira $\mathrm{RH}$, et al. Risk factors and survival impact of primary graft dysfunction after lung transplantation in a single institution. Transplant Proc. 2012;44(8):2462-8. http://dx.doi.org/10.1016/j. transproceed.2012.07.134

11. Diamond JM, Lee JC, Kawut SM, Shah RJ, Localio AR, Bellamy $\mathrm{SL}$, et al. Clinical risk factors for primary graft dysfunction after lung transplantation. Am J Respir Crit Care Med. 2013;187(5):527-34 http://dx.doi.org/10.1164/rccm.201210-18650C

12. Stewart S, Fishbein MC, Snell GI, Berry GJ, Boehler A, Burke MM, et al. Revision of the 1996 working formulation for the standardization of nomenclature in the diagnosis of lung rejection. $J$ Heart Lung Transplant. 2007;26(12):1229-42. http://dx.doi.org/10.1016/j. healun.2007.10.017

13. Todd JL, Palmer SM. Bronchiolitis obliterans syndrome: the fina frontier for lung transplantation. Chest. 2011;140(2):502-8. http:// dx.doi.org/10.1378/chest.10-2838

14. Paraskeva M, McLean C, Ellis S, Bailey M, Williams T, Levvey B, et al. Acute fibrinoid organizing pneumonia after lung transplantation. Am J Respir Crit Care Med. 2013;187(12):1360-8. http://dx.doi. org/10.1164/rccm.201210-18310C

15. Todd JL, Jain R, Pavlisko EN, Finlen Copeland CA, Reynolds JM Snyder LD, et al. Impact of forced vital capacity loss on survival after the onset of chronic lung allograft dysfunction. Am J Respir Crit Care Med. 2014;189(2):159-66.

16. Fishman JA. Infection in solid-organ transplant recipients. $N$ Engl J Med. 2007:357(25):2601-14. http://dx.doi.org/10.1056/ NEJMra064928

17. Samano MN, Minamoto $H$, Junqueira JJ, Yamaçake KG, Gomes $H A$, Mariani AW, et al. Bronchial complications following lung transplantation. Transplant Proc. 2009:41(3):921-6. http://dx.doi. org/10.1016/j.transproceed.2009.01.047

18. de la Torre M, Fernández R, Fieira E, González D, Delgado M Méndez $L$, et al. Postoperative surgical complications after lung transplantation. Rev Port Pneumol (2006). 2015;21(1):36-40.

19. Timrott K, Vondran FW, Kleine M, Warnecke G, Haverich A, Lehner $F$, et al. The impact of abdominal complications on the outcome after thoracic transplantation-a single center experience. Langenbecks Arch Surg. 2014;399(6):789-93. http://dx.doi.org/10.1007/s00423014-1193-7 